\title{
The interplay between wetting and demixing in non-additive symmetric mixtures at selective walls
}

\author{
Andrzej Patrykiejew ${ }^{\mathrm{a}}$ \\ Department for the Modelling of Physico-Chemical Processes, Faculty of Chemistry, MCS University, 20031 Lublin, \\ Poland
}

Received 30 April 2018

Published online 1 October 2018

(C) The Author(s) 2018. This article is published with open access at Springerlink.com

\begin{abstract}
The Monte Carlo simulation method in the grand canonical ensemble has been used to study the wetting behavior of highly non-additive symmetric mixtures in contact with selective walls. We have focused on the behavior of the mixtures, which exhibit closed immiscibility loops in the bulk, and have investigated the interplay between the surface selectivity and the wetting properties of the adsorbed mixtures. Different wetting behaviors has been found, depending on the values of the adsorption energies of the mixture components. We have considered the systems in which the wetting at non-selective walls occurs at the temperatures below the onset of the demixing transition in the bulk. When the average value of adsorption energies is fixed, the wetting temperature has been found to be unaffected by the weak selectivity of the wall. In this case, the reentrant mixing has been observed in thick adsorbed films. However, when the wall selectivity increases beyond a certain threshold value, the reentrant mixing in the film has been suppressed. This leads to the increase of the wetting temperature. In the case, when the adsorption energy of one component is fixed, while the adsorption energy of the second component changes, the wetting temperature has been found to exhibit non-monotonous changes with the difference between the adsorption energies of the mixture components.
\end{abstract}

\section{Introduction}

It has been now well established that a simple model of non-additive symmetric mixtures exhibits complex phase behavior [1-8]. In the binary symmetrical mixture, its pure components are indistinguishable. Thus, the interaction potentials for the $\mathrm{AA}$ and $\mathrm{BB}$ pairs are the same. Only the interaction potential acting between the pairs $\mathrm{AB}$ may have different strength and/or range. Thus, the properties of a symmetric mixture are determined by two parameters. The first is defined by the ratio of the interaction strengths of the $\mathrm{AB}$ and $\mathrm{AA}(\mathrm{BB})$ interactions, $e=\varepsilon_{A B} / \varepsilon_{A A}$, and the second is defined by the ratio of the parameters which define the ranges of the interaction between different pairs, $s=\sigma_{A B} / \sigma_{A A}$. The parameter $e$ measures the energetic non-additivity, and the parameter $s$ is a measure of the geometric non-additivity. The phase behavior of symmetric mixtures has been found to change, depending on the magnitudes of these two parameters. When $e<1$, the energy of interaction between the like particles is higher that the energy of interaction between the pairs of unlike particles, and the mixture is expected to undergo a demixing transition. However, the demixing transition has been found to be also strongly affected by

\footnotetext{
${ }^{\mathrm{a}}$ e-mail: andrzej ·patrykiejew@gmail .com
}

the magnitude of the parameter $s$. In general, the values of $s$ higher than unity enhance demixing, while the values of $s$ lower than unity suppress demixing. Such situation results from the competing energetic and entropic (packing) effects. The demixing transition has been observed to occur in the liquid and/or in the solid phases, and to be of the first or higher order, depending again on the magnitudes of the parameters $e$ and $s$, as well as on the temperature.

In particular, it has been demonstrated [8] that when the parameters $e$ and $s$ are both considerably smaller than unity, the symmetric mixtures may exhibit closed immiscibility loops in the liquid phase. In such cases, the vapor condenses into the mixed liquid at low temperatures, since the packing effects are strong. The mixed liquid can attain much higher density than the demixed liquid. At very low temperatures, the ratio of average number densities of a perfectly mixed and demixed solid phases is roughly proportional to $s^{-3}$. On the other hand, the ratio of interaction energies in perfectly mixed and demixed solid phase is proportional to $s^{-6} e$. Of course, these relations cannot be directly applied to liquids. Nonetheless, they indicate that the packing effects can quite successfully compete with the energetic effects, when the parameter $s$ is sufficiently small. For example, it has been shown [8] that when $e=0.6$, the mixture with $s=0.6$ does not show any trace 


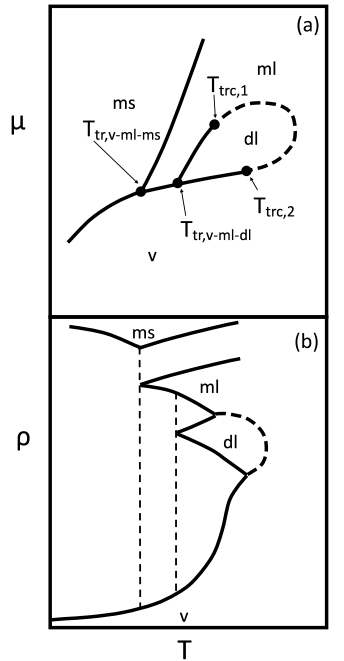

Fig. 1. The schematic representations of the bulk phase diagram for the mixture with $e=0.6$ and the values of the parameter $s$ between 0.7 and 0.74 . Parts a and b show $\left(T^{*}, \mu^{*}\right)$ and $\left(T^{*}, \rho\right)$ projections, respectively. The regions corresponding to the vapor, the mixed liquid, the demixed liquid and the mixed solid phases are marked by $\mathrm{v}, \mathrm{ml}$, dl and ms, respectively. $T_{t r c, 1}$ and $T_{t r c, 2}$ mark the tricritical points, and $T_{t r, v-m l-m s}$ and $T_{t r, v-m l-d l}$ correspond to the different triple points. The first-order phase boundaries are given by solid lines, and the dashed line corresponds to the $\lambda$-lines.

of phase separation in the liquid, while the mixtures with $s>0.7$ undergo the demixing transition, and the temperature at which the demixing transition occurs gradually decreases with $s$.

It has been shown that the behavior of non-uniform symmetric mixtures is also very complex. The wetting behavior of only energetically non-additive symmetric mixtures, when $e<1$, and $s=1$, in contact with nonselective solid substrates has been studied by several authors [9-16]. The assumption that $s=1$ has allowed to apply different theoretical approaches [11,13-15], including the density functional theory and lattice gas models. These methods cannot be applied to symmetric mixtures with negative geometrical non-additivity $(s<1)$.

Also, the literature describing the behavior of symmetric mixtures confined in pores is quite abundant [17-24].

It is worth noting that symmetric mixtures can be treated as a simple model of racemic mixtures [25], which consist of $\mathrm{D}$ and $\mathrm{L}$ isomers. The separation of racemic mixtures into the pure $\mathrm{D}$ and $\mathrm{L}$ isomers is of great importance in several fields, like biology and drug industry, since the $\mathrm{D}$ and L enantiomers very often show different biological activity [26]. The separation of racemic mixtures can be achieved in a number of ways, and several of them involve adsorption at chiral surfaces $[27,28]$. For example, kinked-stepped, high Miller index surfaces of metal crystals are chiral and exhibit enantiospecific properties [29]. In particular, the adsorption energies of D and L isomers on such naturally chiral surfaces are different [30]. Using the concept of a symmetric mixture, it is possible to model adsorption on chiral surfaces, by assuming that the mixture components interact differently with the solid surface. Although the model of spherical non-additive symmetric mixtures is very simple, nevertheless, it can shed a new light on the interplay between the properties of bulk mixtures and their wetting behavior.

The wetting of non-selective surfaces by energetically $(e<1)$ and geometrically $(s \neq 1)$ non-additive symmetric mixtures have also been studied, but only by the computer simulation methods [31,32]. In the special case of only geometrically non-additive mixtures $(e=1$ and $s \neq 1)$, which do not phase separate, the wetting behavior has been found to depend strongly on the structure of the mixed bulk liquid and solid phases [31]. It has been demonstrated that for a given strength of the surface potential, the wetting temperature shows non-monotonous changes with $s$, following the changes of the bulk triple point temperature. This occurs even when the wetting temperature is located well above the bulk triple point, and indicates that the short range ordering in the liquid-like films and in the bulk liquids plays an important role in the wetting phenomena.

The study of wetting behavior of energetically and geometrically non-additive mixtures, with $e<1$ and $s<1$, at non-selective walls, has concentrated on the systems, which exhibit closed immiscibility loops in the bulk [32]. In such cases, the vapor condenses into the mixed liquid at low temperatures, and into the demixed liquid at the temperatures above the triple point, $T_{t r, v-m l-d l}$, in which the vapor coexists with the mixed and demixed liquids. These mixtures show the phase diagrams like that given in Figure 1. Since the AB interaction is weaker than the AA and $\mathrm{BB}$ interactions $(e<1)$, the mixed adsorbed film experiences a relatively stronger attraction to the surface than the demixed film. The attractive surface always increases the tendency towards mixing in the film, and the mixing in the film is also enhanced by the negative geometrical non-additivity $(s<1)$. In such cases, the $\mathrm{AB}$ pairs are preferred in particular when the density is high. These two effects lead to the stabilization of mixed films, even when the chemical potential approaches the bulk coexistence, and the temperature is higher than $T_{t r, v-m l-d l}$. When it happens, only a partial wetting takes place at the temperature of the triple point $T_{t r, v-m l-d l}$ and above, since the mixed film cannot grow to form a macroscopic (mixed) liquid layer. The bulk liquid becomes demixed at $T \geq T_{t r, v-m l-d l}$, and the stable mixed liquid appears only at the higher chemical potential, at which the demixed and mixed liquids coexist (see Fig. 1). However, taking into account that a complete wetting is bound to appear at a certain temperature below the temperature at which the vapor-liquid coexistence terminates [33,34], the systems considered in reference [32] have been found to show two, upper and lower wetting transitions.

In this work, we have considered the wetting behavior of symmetric mixtures in contact with selective walls. The primary aim of our study has been to elucidate how the wetting behavior of highly non-additive symmetric mixtures changes, when the bulk mixtures show different tendency towards mixing in the bulk. In particular, we have concentrated on the mixtures with the bulk phase 
diagram like that shown in Figure 1. It should be mentioned that we have already discussed the effects of wall selectivity on the wetting of the mixture with $e=0.6$ and $s=0.73$ [35], which also show the closed immiscibility loop. Here, we have considered the mixtures of the lower tendency towards demixing, with $s=0.7$ and 0.72 , and demonstrated how the wall selectivity affects their wetting behavior.

\section{The model and simulation method}

The model applied here has been the same as that used in reference [35]. Thus, the potential describing the interaction between the components of the binary symmetric mixture, consisting of the components $\mathrm{A}$ and $\mathrm{B}$, has been represented by the truncated $(12,6)$ Lennard-Jones potential

$$
u_{i j}(r)= \begin{cases}4 \varepsilon_{i j}\left[\left(\sigma_{i j} / r\right)^{12}-\left(\sigma_{i j} / r\right)^{6}\right] & r \leq r_{\max } \\ 0 & r>r_{\max }\end{cases}
$$

where $r$ is the distance between a pair of the particles $i$ and $j$. We have taken the value of $\varepsilon_{A A}=\varepsilon_{B B}=\varepsilon$ as the unit of energy, and $\sigma_{A A}=\sigma_{B B}=\sigma$ as the unit of length. Therefore, the parameters, $e=\varepsilon_{A B} / \varepsilon_{A A}$ and $s=\sigma_{A B} / \sigma_{A A}$, characterize non-additivity of the bulk fluid. We have also introduced the reduced temperature, $T^{*}=k T / \varepsilon$, and the reduced chemical potentials of the species $A$ and $B, \mu_{i}^{*}=\mu_{i} / \varepsilon, i=A, B$. All the energy like quantities have been expressed in the units of $\varepsilon$. The fluid-fluid interaction potential has been cut at the distance $r_{\max }^{*}=3.0$. Throughout this paper we have assumed that $\mu_{A}^{*}=\mu_{B}^{*}=\mu^{*}$.

The mixture has been placed in contact with a selective smooth wall. The fluid-wall interaction potential, being a function of the distance from the wall $(z)$ only, has been represented by the following equation:

$$
v_{i}(z)=\varepsilon_{g s, i}^{*}\left[\left(\frac{\sigma}{z}\right)^{9}-\left(\frac{\sigma}{z}\right)^{3}\right]
$$

In the above $\varepsilon_{g s, i}^{*}$ is a measure of the fluid-wall interaction strength (expressed in the units of $\varepsilon$ ) for the $i$-the component ( $\mathrm{A}$ or $\mathrm{B}$ ). The fluid-wall interaction potential has been cut at the distance from the surface equal to $z_{\max }^{*}=10$.

The model has been studied using the Monte Carlo simulation method in the grand canonical ensemble $[31,32,36-38]$. Using the cells of the size $L_{x}^{*} \times L_{y}^{*} \times L_{z}^{*}=$ $20 \times 20 \times 60$ the standard periodic boundary conditions in the $x$ and $y$ directions have been applied. The solid surface has been placed at $z=0$ and the simulation box has been closed from the top, at $z=L_{z}^{*}$, by a reflecting hard wall.

The quantities recorded included the average numbers of particles $A\left(N_{A}\right)$ and $B\left(N_{B}\right)$ and the density profiles for each component, $\rho_{k}(z)$. The density profiles allow to calculate the surface excess densities of the components, $\rho_{e x, k}$, using the following equation

$$
\rho_{e x, k}=\frac{1}{S} \int_{0}^{L_{z}}\left[\rho_{k}(z)-\rho_{o, k}\right] d z
$$

where $S$ is the surface area, and $\rho_{o, k}$ is the bulk density of the $k$-th component. The total excess density has been obtained as a sum $\rho_{e x}=\rho_{e x, A}+\rho_{e x, B}$.

Using the density profiles, we have also calculated the order parameter profiles

$$
m(z)=\frac{\left|\rho_{A}(z)-\rho_{B}(z)\right|}{\rho_{A}(z)+\rho_{B}(z)}
$$

being the measure of phase separation across the adsorbed film.

Besides, we have calculated the total order parameter $m$, defined as

$$
m=\frac{\left|\rho_{e x, B}-\rho_{e x, A}\right|}{\rho_{e x}} .
$$

Of course, we have also monitored the average fluidfluid potential energy (per particle), $\left\langle u_{g g}^{*}\right\rangle$, the average fluid-wall potential energies for both components $\left\langle u_{g s, k}^{*}\right\rangle$ ( $k=A$ or $B$ ), and the total potential energy of the system.

The wetting behavior has been investigated using the excess adsorption isotherms calculated at different temperatures, and the density profiles recorded at different temperatures and at different values of the chemical potential. The phase boundaries of the bulk systems have been taken from references [31,32]. We have performed the runs involving $10^{8}-5 \times 10^{9}$ Monte Carlo steps. Each Monte Carlo step consisted of randomly chosen attempts to translate a randomly chosen particle, to create a new particle in a randomly chosen position, to annihilate a randomly chosen particle or to change the identity of a randomly chosen particle. Similar numbers of Monte Carlo steps have been used to equilibrate the system.

Throughout this paper, we have considered the mixtures with the parameter $e$ equal to 0.6 , and the parameter $s$ equal to 0.70 and 0.72 . It has already been mentioned in Section 1 that the bulk phase diagrams of these mixtures are qualitatively the same $[31,32]$ (see Fig. 1), and that these mixtures exhibit closed immiscibility loops. At low temperatures, between the triple point $T_{t r, v-m l-m s}^{*}$, in which the vapor coexists with the mixed liquid and the mixed solid, and the triple point $T_{t r, v-m l-d l}^{*}$, in which the vapor coexists with the mixed and demixed liquids, the vapor condenses into the mixed liquid. Only at the temperatures exceeding the triple point $T_{t r, v-m l-d l}^{*}$, the demixing transition accompanies the vapor condensation. It should be noted that the demixing is the first order transition at the temperatures between $T_{t r, v-m l-d l}^{*}$ and the tricritical point $T_{t r c, 1}^{*}$. The tricritical point $T_{t r c, 1}^{*}$ is the onset of the so-called $\lambda$-line, along which the demixing occurs via the continuous transition. The vapor-liquid coexistence terminates at still another tricritical point, $T_{t r c, 2}^{*}$, which replaces the usual 
vapor-liquid critical point, and being also the onset of $\lambda$-line. Thus, the $\lambda$-line forms a closed loop, as shown by the dashed line in Figure 1.

\section{Results and discussion}

To begin with, we present the results for the mixture with $s=0.7$. In this case, the onset of the demixing transition is located at the triple point temperature of about $T_{t r, v-m l-d l}^{*}=1.11$, which is quite close to the tricritical point $T_{t r c, 2}^{*} \approx 1.18$. It has been demonstrated [32] that in the case of non-selective walls, with $\varepsilon_{g s, A}^{*}=\varepsilon_{g s, B}^{*}=\varepsilon_{g s}^{*}$, this mixture shows two wetting points. The lower wetting transition occurs at the temperature $T_{w, 1}^{*}$, below $T_{t r, v-m l-d l}^{*}$, when the developing mixed wetting film and the bulk liquid are mixed. Of course, the wetting temperature decreases when $\varepsilon_{g s}^{*}$ becomes larger, and for $\varepsilon_{g s}^{*}$ equal to and higher than about 6 , the wetting has been found to be pinned in the triple point $T_{t r, v-m l-m s}^{*} \approx 0.865$, in which the vapor coexists with the mixed liquid and the mixed solid phases. However, a complete wetting ceases as soon as the temperature reaches the triple point $T_{t r, v-m l-d l}^{*}$, because the adsorbed film remains mixed up to the bulk coexistence. This is a consequence of the enhanced mixing in the film due to the attractive fluidsurface potential. However, a complete wetting is bound to reappear below the tricritical point $T_{t r c, 2}^{*}$, as it follows from the theoretical argument of Cahn [33] and of Ebner and Saam [34]. These authors have demonstrated that a complete wetting has to occur at the temperature lower than the vapor-liquid critical point. The same applies to the systems in which the vapor-liquid coexistence terminates in the tricritical point. This implies the presence of the second (upper) wetting transition above $T_{t r, v-m l-d l}^{*}$, but below $T_{t r c, 2}^{*}$. Indeed, we have found a rather clear evidence of such behavior [32]. The situation is expected to be different when the mixture is in contact with selective walls.

The first series of calculations has been carried out for the fixed mean value of the surface potential strengths of the components, $\bar{\varepsilon}_{g s}^{*}=(1 / 2)\left(\varepsilon_{g s, A}^{*}+\varepsilon_{g s, B}^{*}\right)=5.5$. The lower wetting temperature $\left(T_{w, 1}^{*}\right)$ of the system with $\Delta \varepsilon_{g s}^{*}=\varepsilon_{g s, A}^{*}-\varepsilon_{g s, B}^{*}=0$ (a non-selective wall) has been estimated to be equal to about $T_{w}^{*} \approx 0.92$. This temperature is considerably lower then the triple point temperature $T_{t r, v-m l-d l}^{*}$. Our aim here has been to find, how the increasing difference between $\varepsilon_{g s, A}^{*}$ and $\varepsilon_{g s, B}^{*}$ affects the wetting behavior. Obviously, when $\Delta \varepsilon_{g s}^{*}$ increases, the adsorbed film has to show a growing tendency towards demixing, due to the preferential adsorption of one component.

In the limit of very low adsorption, i.e., in the Henry's low region, when one can assume that the adsorbed molecules do not interact one with another, the surface excesses of the components $\mathrm{A}$ and $\mathrm{B}$ are independent of $s$ and $e$, and depend only on the magnitudes of $\varepsilon_{g s, A}^{*}$ and $\varepsilon_{g s, B}^{*}$. Namely, the surface excess of the $k$ th component is given by the following equation

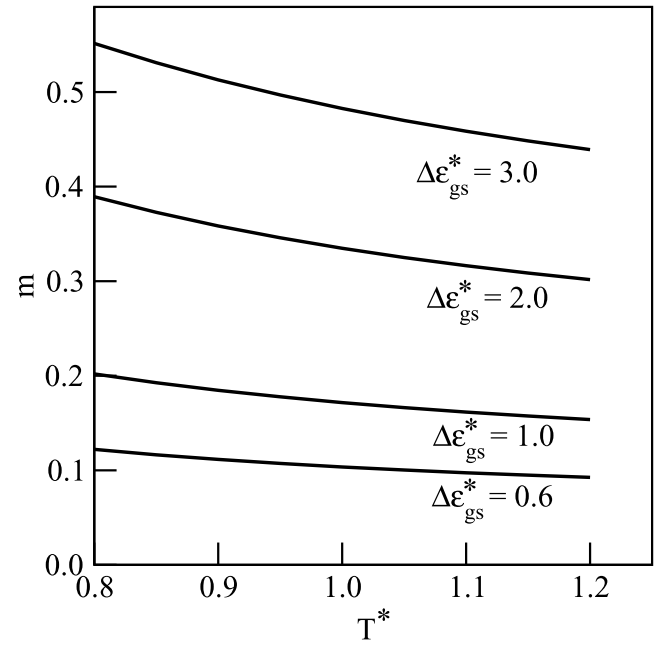

Fig. 2. The changes of the order parameter $m$ versus temperature in the low adsorption limit (in the Henry's region) for $\left\langle\varepsilon_{g s}^{*}\right\rangle=5.5$ and different values of $\Delta \varepsilon_{g s}^{*}$.

$$
\rho_{e x, k} \sim \rho_{o, k} \int_{z_{o}}^{\infty}\left[\exp \left[-v_{k}^{*}\left(z^{*}\right) / T^{*}\right]-1\right] d z
$$

In the above, the integration starts at the Gibbs dividing surface located at $z_{0}$. The bulk densities of the components $\mathrm{A}$ and $\mathrm{B}$ are bound to be same, since their chemical potentials are equal. The surface excesses, $\rho_{e x, A}$ and $\rho_{e x, B}$, allow to calculate the order parameter $m$ (cf. Eq.(5)). In Figure 2 we have shown the changes of the order parameter $m$ with temperature, for several choices of $\Delta \varepsilon_{g s}^{*}$ and for $\bar{\varepsilon}_{g s}^{*}=5.5$. An increase of $\Delta \varepsilon_{g s}^{*}$ leads to the enhancement of demixing, i.e., to the preferential adsorption of the component B. This figure also shows that the degree of demixing gradually decreases with temperature, as expected.

When the film density becomes larger, one has to take into account the interaction between the adsorbed particles, and the behavior of the growing film has to change. We have calculated the excess adsorption-desorption isotherms for several choices of $\Delta \varepsilon_{g s}^{*}$, between 0.2 and 2.6 , and at different temperatures. The examples of our results have been presented in Figures 3-7. For the values of $\Delta \varepsilon_{g s}^{*}$ up to 1.0, we have found the same behavior as in the case of the non-selective wall $\left(\Delta \varepsilon_{g s}^{*}=0.0\right)$ with $\varepsilon_{g s, A}^{*}$ and $\varepsilon_{g s, B}^{*}$ equal to 5.5 [32]. In particular, the wetting temperature has been found to occur at $T_{w}^{*} \approx 0.92$. The main part of Figure 3 presents the examples of the total surface excess adsorption isotherms, recorded for the system with $\Delta \varepsilon_{g s}^{*}=1.0$, at different temperatures, right at and above the wetting temperature. It is evident that thick wetting films develop. The inset in Figure 3 shows the changes of the order parameter $m$ along the isotherms and demonstrates that at the temperatures below the triple point $T_{t r, v-m l-d l}^{*} \approx 1.11$, the films undergo a reentrant mixing when the chemical potential approaches the bulk coexistence. However, the situation changes when the temperature exceeds the triple point $T_{t r, v-m l-d l}^{*}$. Under 


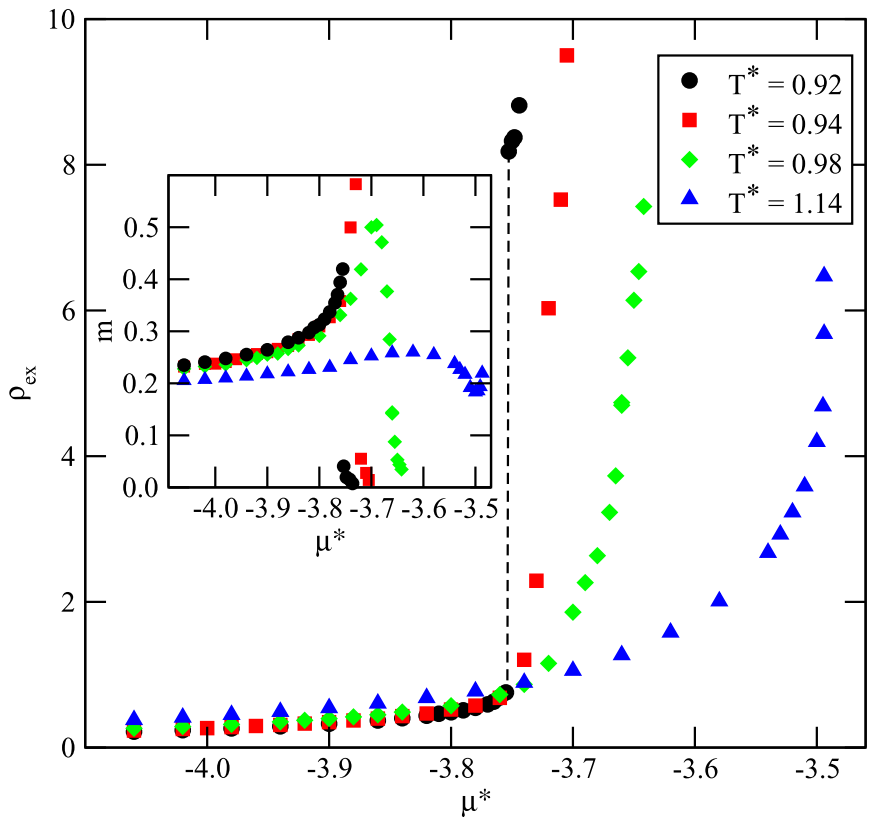

Fig. 3. The main figure shows the excess adsorption isotherms $\left(\rho_{\text {ex }}\right.$ versus $\left.\mu^{*}\right)$, while the inset shows the corresponding changes of the order parameter $m$ with the chemical potential $\mu^{*}$ at different temperatures (given in the figure), for the system with $\left\langle\varepsilon_{g s}^{*}\right\rangle=5.5$ and $\Delta \varepsilon_{g s}^{*}=1.0$.

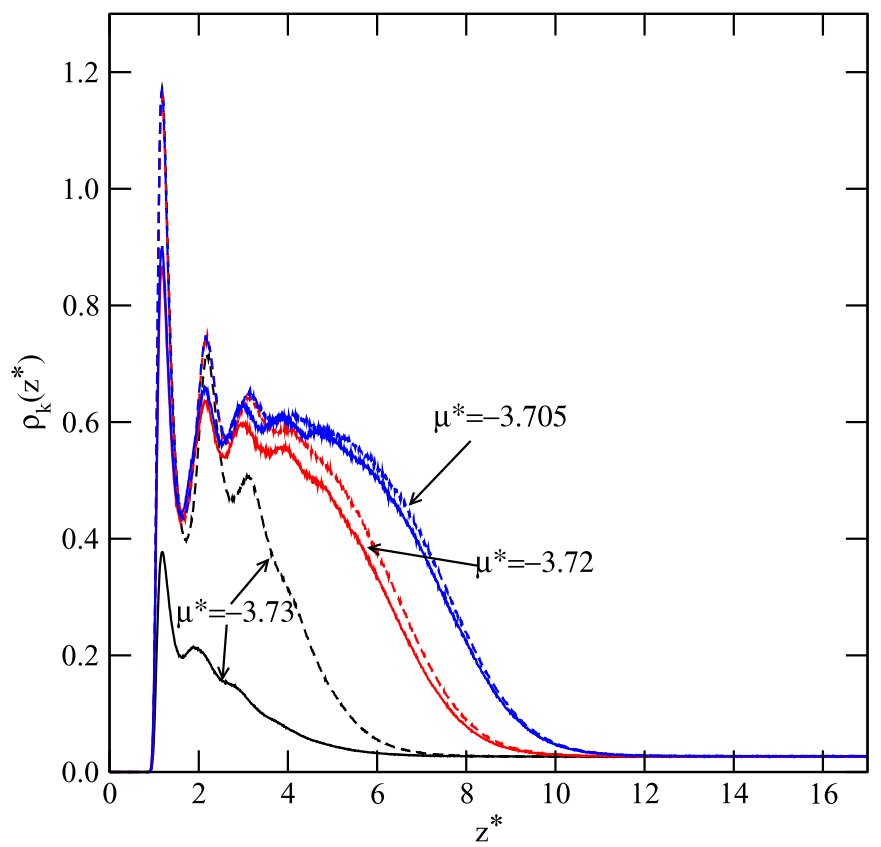

Fig. 4. The examples of density profiles for the components A (solid lines) and $\mathrm{B}$ (dashed lines) for the system with $e=0.6$, $s=0.70,\left\langle\varepsilon_{g s}^{*}\right\rangle=5.5$ and $\Delta \varepsilon_{g s}^{*}=1.0$ at $T^{*}=0.94$ and different values of the chemical potential (shown in the figure). The color key applies to the on-line version of the paper.

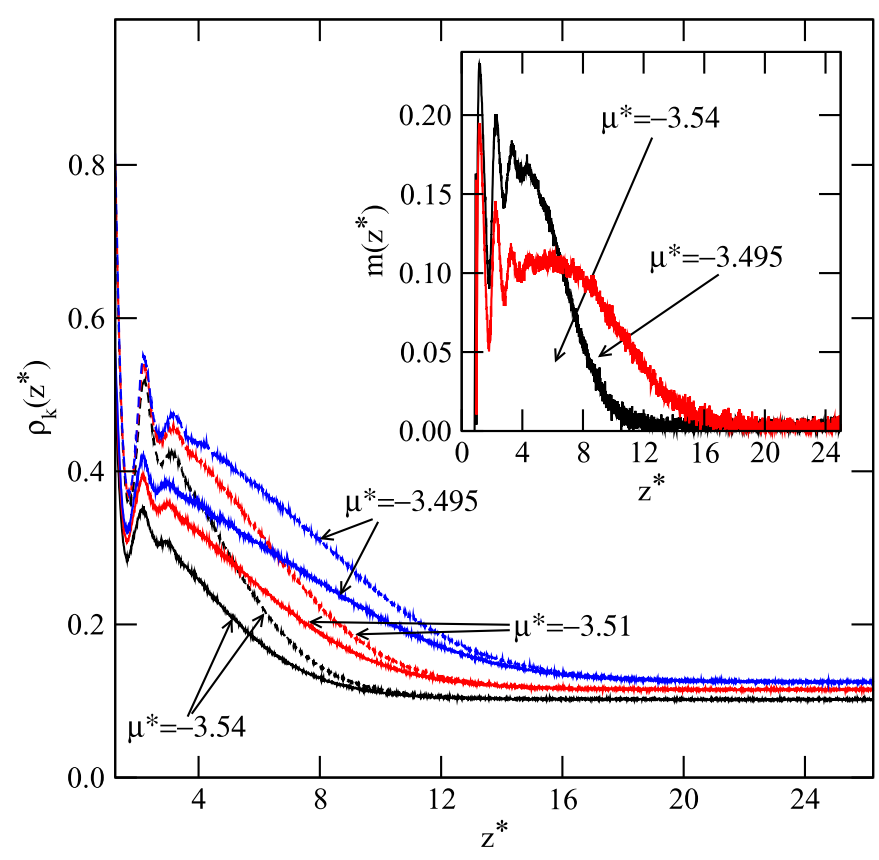

Fig. 5. The examples of density profiles for the components A (solid lines) and $\mathrm{B}$ (dashed lines) for the system with $e=0.6$, $s=0.70,\left\langle\varepsilon_{g s}^{*}\right\rangle=5.5$ and $\Delta \varepsilon_{g s}^{*}=1.0$ at $T^{*}=1.14$ and different values of the chemical potential (shown in the figure). The color key applies to the on-line version of the paper. The inset gives the order parameter profiles for two values of $\mu^{*}$.

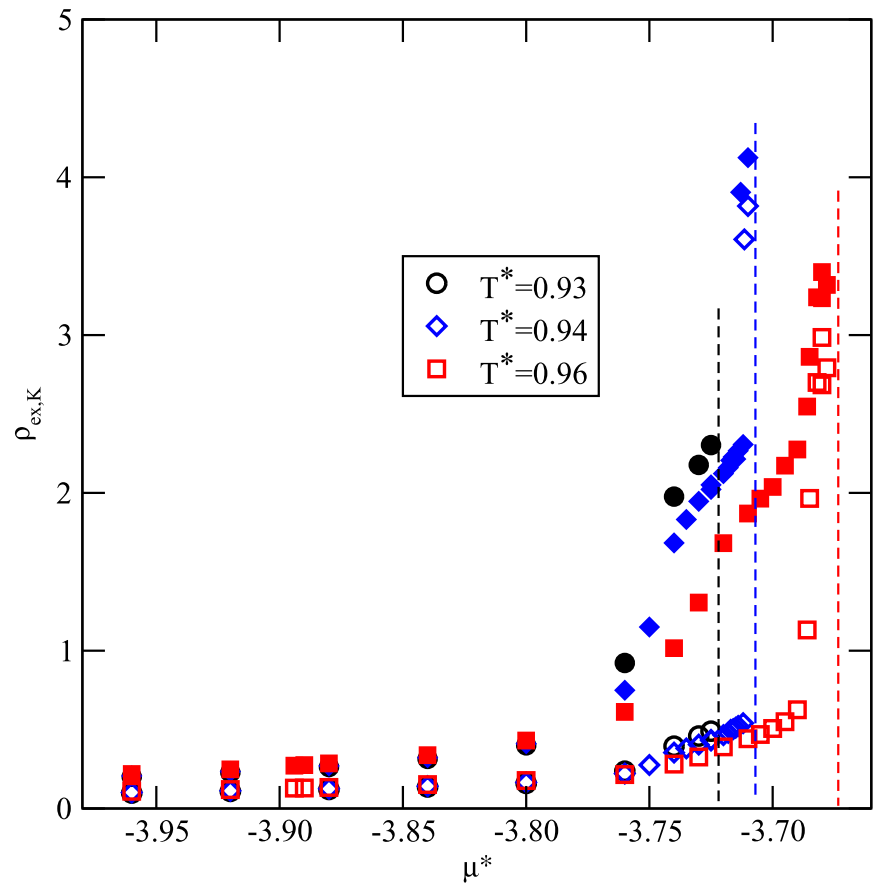

Fig. 6. The excess adsorption isotherms $\rho_{e x, K}$ of the components $K=A$ (open symbols) and $K=B$ (filled symbols) at $T^{*}=0.93,0.94$ and 0.96 , for the system with $\left\langle\varepsilon_{g s}^{*}\right\rangle=5.5$ and $\Delta \varepsilon_{g s}^{*}=1.4$. 


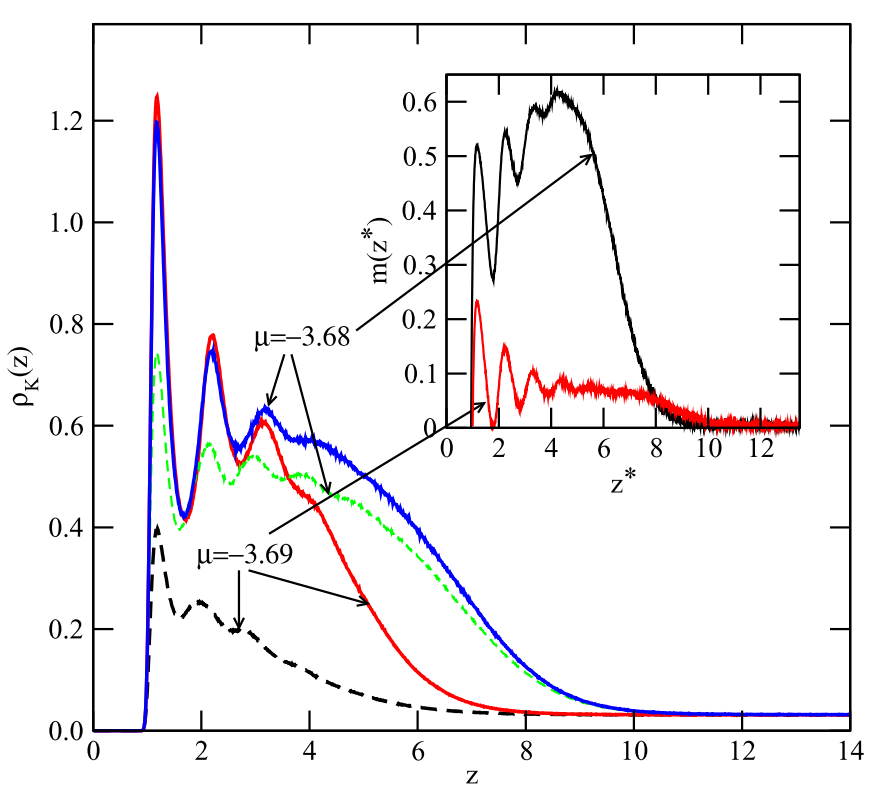

Fig. 7. The main figure shows the density profiles of the components A (dashed lines) and B (solid lines) recorded for the system with $\left\langle\varepsilon_{g s}^{*}\right\rangle=5.5$ and $\Delta \varepsilon_{g s}^{*}=1.4$ at $T^{*}=0.96$ and different values of the chemical potential (given in the figure). The inset shows the corresponding order parameter profiles.

such condition, the vapor condenses into the demixed liquid, and the order parameter starts to increase when $\mu^{*}$ approaches the bulk coexistence (see the order parameter changes at $T^{*}=1.14$ in the inset to Fig. 3). We have not been able to get very close to the bulk coexistence point, due to the finite size of the simulation cell.

The reentrant mixing in the film has also been illustrated by the density profiles shown in Figure 4, recorded for the same system, at $T^{*}=0.94$ and different values of the chemical potential. The thin film (at $\left.\mu^{*}=-3.73\right)$ is highly demixed, while the thick film is mixed and attains a rather high density. Only the first two layers, adjacent to the surface, exhibit a substantial enrichment in the more strongly adsorbed component B. On the other hand, at $T^{*}>T_{t r, v-m l-d l}^{*}$, the density profiles of both components (see the main part of Fig. 5) show large differences over the entire film. The profiles given in Figures 4 and 5 also show that the interface between the film and the bulk vapor becomes more and more diffused when the temperature increases. The inset in Figure 5 shows the order parameter profiles at the values of $\mu^{*}$ rather close to the bulk coexistence and demonstrates that a partial demixing occurs even in the outer parts of the adsorbed layer.

An increase of $\Delta \varepsilon_{g s}^{*}$ above 1.0 leads to the changes in the wetting behavior. The predominant adsorption of the component $\mathrm{B}$ increases and causes a stronger phase separation in the adsorbed layer. The film may remain demixed at the bulk coexistence, at higher temperatures than observed for smaller values of $\Delta \varepsilon_{g s}^{*}$. This implies that the wetting temperature should also increase. For example, we have found that when $\Delta \varepsilon_{g s}^{*}=1.4$, the reentrant mixing in the film does not occur at $T^{*}=0.94$, but it is present at higher temperatures. This is illustrated by the

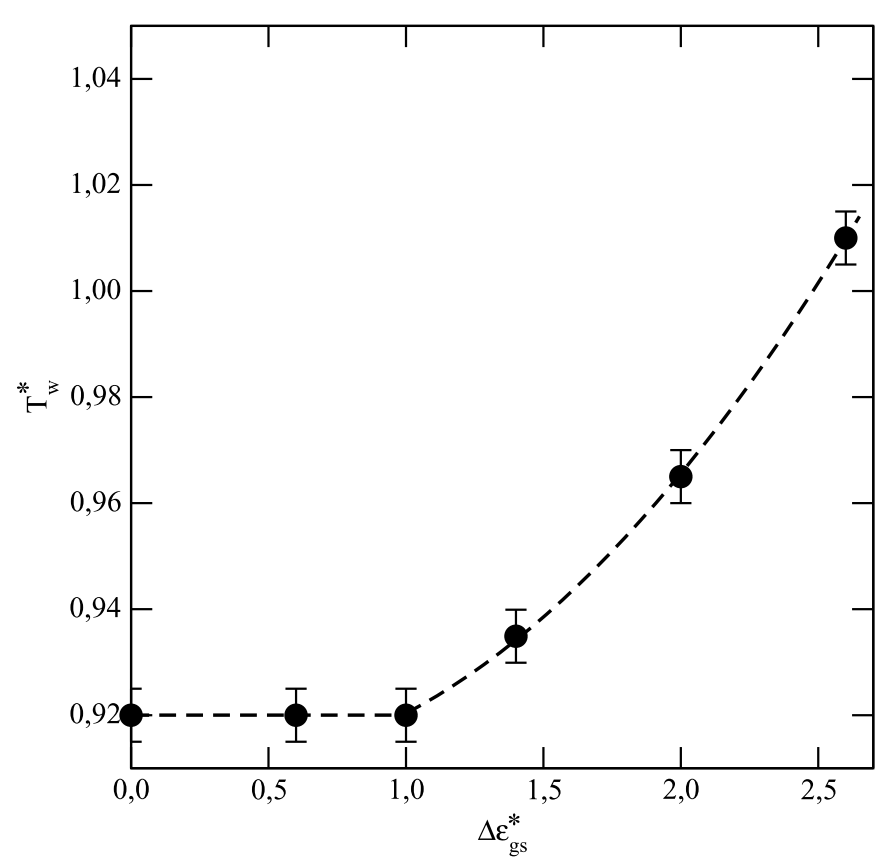

Fig. 8. The changes of the wetting temperature against the difference between the adsorption energies of the components $\left(\Delta \varepsilon_{g s}^{*}\right)$ for the systems with $s=0.70$ and $\left\langle\varepsilon_{g s}^{*}\right\rangle=5.5$.

excess adsorption isotherms of the mixture components, depicted in Figure 6. At $T^{*}=0.93$ and 0.94 the adsorbed films remain demixed and rather thin, while the reentrant mixing occurs at higher temperatures, and the film attains larger thickness. The reentrant mixing is accompanied by a large decrease of the order parameter $m$ in the entire film (see the inset to Fig. 7). However, the density profiles given in the main part of Figure 7 show that the reentrant mixing causes the density of the more strongly adsorbed component $\mathrm{B}$ to undergo large changes only in the outer part of the film, while the component A shows a large increase of density in the entire film.

We have also carried out simulation for still larger values of $\Delta \varepsilon_{g s}^{*}$, up to 2.6, and the results are summarized in Figure 8, which presents the changes of the wetting temperature with $\Delta \varepsilon_{g s}^{*}$. It is quite clear that as long as $\Delta \varepsilon_{g s}^{*} \leq 1.0$ the wetting temperature does not change, and then rather sharply increases when $\Delta \varepsilon_{g s}^{*}$ becomes greater. Upon the increase of $\Delta \varepsilon_{g s}^{*}$, the stability of the demixed films also increases. Only at sufficiently high temperatures, the entropic effects become large enough to trigger the reentrant mixing, which allows for a complete wetting.

The second series of calculations has been carried out assuming the fixed value of $\varepsilon_{g s, A}^{*}=5.0$, and different, greater than $\varepsilon_{g s, A}^{*}$, values of $\varepsilon_{g s, B}^{*}$, between 5.2 and 8.0. We recall that when $\varepsilon_{g s, A}^{*}$ and $\varepsilon_{g s, B}^{*}$ are both equal to 5.0 (a non-selective wall), the wetting occurs below $T_{t r, v-m l-d l}^{*}$, at the wetting temperature $T_{w, 1} \approx 0.97$. In the previous work [35], we have presented the results for a similar situation, but for the mixture characterized by $s=0.73$. It has been shown that when the wetting transition at non-selective wall occurs at the temperature between $T_{t r, v, m l-m s}^{*}$ and $T_{t r, v-m l-d l}^{*}$, an increase of 


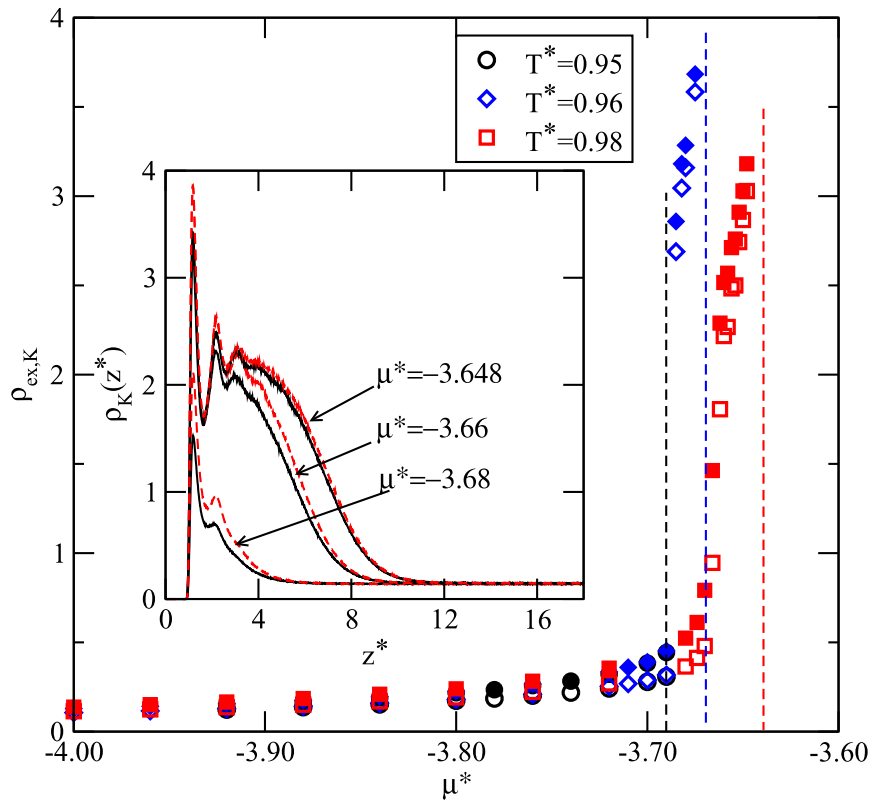

Fig. 9. The main part show, the excess adsorption isotherms of individual components $\left(\rho_{e x, K}\right.$ for the system with $\varepsilon_{g s, A}^{*}=5.0$ and $\varepsilon_{g s, B}^{*}=5.4$, at different temperatures. The inset shows the density profiles for the components A (solid lines) and $\mathrm{B}$ ( dashed lines) at $T^{*}=0.98$ and for different values of the chemical potential (given in the figure).

$\Delta \varepsilon_{g s}^{*}$ leads to non-monotonous changes of $T_{w, 1}^{*}$, and that a complete wetting also occurs at any temperature exceeding $T_{t r, v-m l-d l}^{*}$. The presently considered mixture, with $s=0.7$, shows a considerably stronger tendency towards mixing, and hence its wetting behavior is also expected to be different.

In the main part of Figure 9, we have shown the excess adsorption isotherms at $T^{*}=0.95,0.96$ and 0.98 , recorded for the system with $\Delta \varepsilon_{g s}^{*}=0.4$. One readily notes that at $T^{*}=0.95$, the surface excesses of both components remain quite small, even when the chemical potential approaches the bulk coexistence. On the other hand, at $T^{*}=0.96$, and at the higher temperature of 0.98 , thick mixed films develop. In the case of $T^{*}=0.96$, we have found a sharp prewetting transition, while at $T^{*}=0.98$ the increase of the surface excesses is smooth, implying that the prewetting critical temperature is lower than 0.98. The inset in Figure 9 gives the density profiles of the components $\mathrm{A}$ and $\mathrm{B}$ at $T^{*}=0.98$, and for different values of the chemical potential. It is quite well seen that the film shows a nearly complete mixing upon the approach to the bulk coexistence. Only the first layer exhibits a small enrichment in the component $B$. The above results suggest that the wetting temperature occurs at $T_{w}^{*} \approx 0.955 \pm 0.005$. A very similar behavior has been observed for $\Delta \varepsilon_{g s}^{*}$ up to about 1.4. Only the wetting temperature has been found to gradually decrease.

However, when the difference between $\varepsilon_{g s, A}^{*}$ and $\varepsilon_{g s, B}^{*}$ becomes larger than about 1.4, the wetting temperature has been found to gradually increase (see Fig. 10). In the region of $\Delta \varepsilon_{g s}^{*} \leq 1.4$, the increase of $\varepsilon_{g s, B}^{*}$ causes the

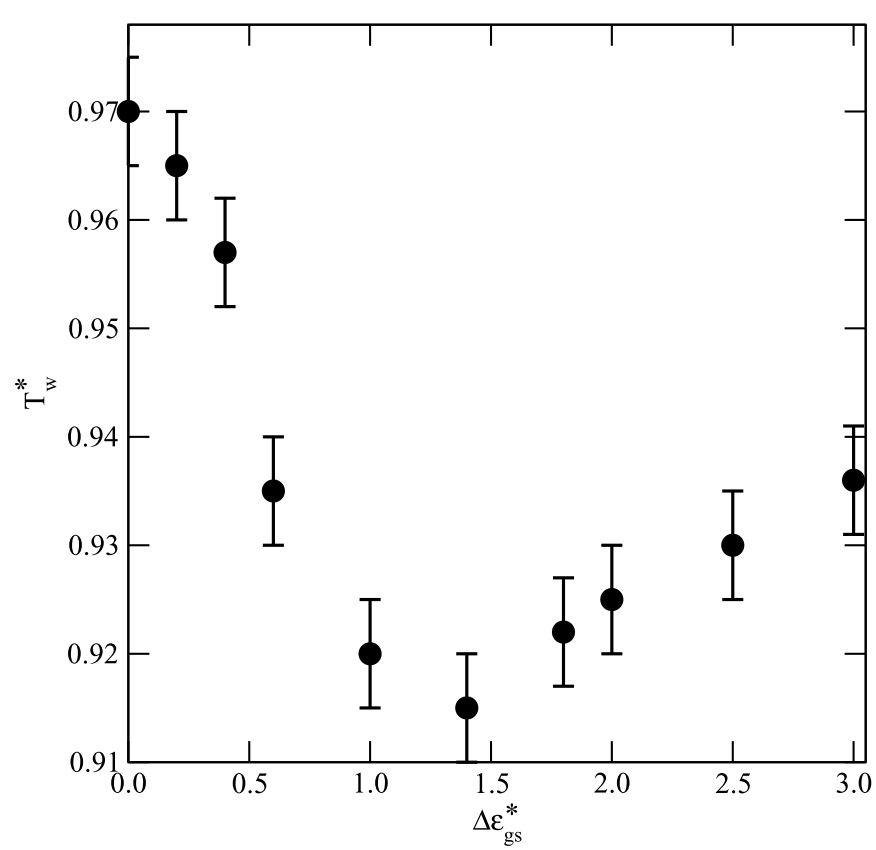

Fig. 10. The changes of the wetting temperature against the difference between the adsorption energies of the components $\left(\Delta \varepsilon_{g s}^{*}\right)$ for the systems with $s=0.70$ and $\varepsilon_{g s, A}^{*}=5.0$.

increase of the average fluid-solid interaction energy in the film, which results in the increase of the film density. This, in turn, favors mixing and leads to a gradual decrease of the wetting temperature. On the other hand, when $\Delta \varepsilon_{g s}^{*}$ becomes high enough, a strong preferential adsorption of the component B stabilizes the demixed film. However, an increase of $\Delta \varepsilon_{g s}^{*}$ is accompanied the increase of the average fluid-solid interaction energy, and therefore the wetting temperature only weakly increases with $\Delta \varepsilon_{g s}^{*}$.

Figure 11 presents a comparison of the fluid-surface interaction energy profiles (upper panels), and the density profiles (lower panels) recorded at the same temperature $T^{*}=0.92$ and the same chemical potential close to the bulk coexistence, $\mu^{*}=-3.75$, for the systems characterized by different values of $\Delta \varepsilon_{g s}^{*}$. In the upper panels of Figure 11, we have also put the values of the average fluid-surface potential energy, calculated as

$$
\left\langle u_{g s}^{*}\right\rangle=\frac{1}{S} \int_{z_{o}}^{L_{z}}\left[\rho_{A}(z) v_{A}(z)+\rho_{B}(z) v_{B}(z)\right] d z .
$$

When $\Delta \varepsilon_{g s}^{*}=0.6$, the wetting temperature is higher than 0.92 , and only a thin adsorbed layer of low density appears. The profiles of $u_{g s, K}^{*}\left(z^{*}\right)$ show that the fluidsolid interactions of both components are not very high. Also, the average fluid-solid potential energy is quite small. Of course, the fluid-solid interaction of the more strongly adsorbed component B is larger. A small enrichment of the film in the component $\mathrm{B}$ is due to a rather small value of $\Delta \varepsilon_{g s}^{*}$. In the case of $\Delta \varepsilon_{g s}^{*}=1.4$, the wetting temperature is lower than 0.92 , and a thick mixed film of much higher density develops. In particular, the layers adjacent to the surface reach very high density, and also show a considerably stronger interaction with the surface. 


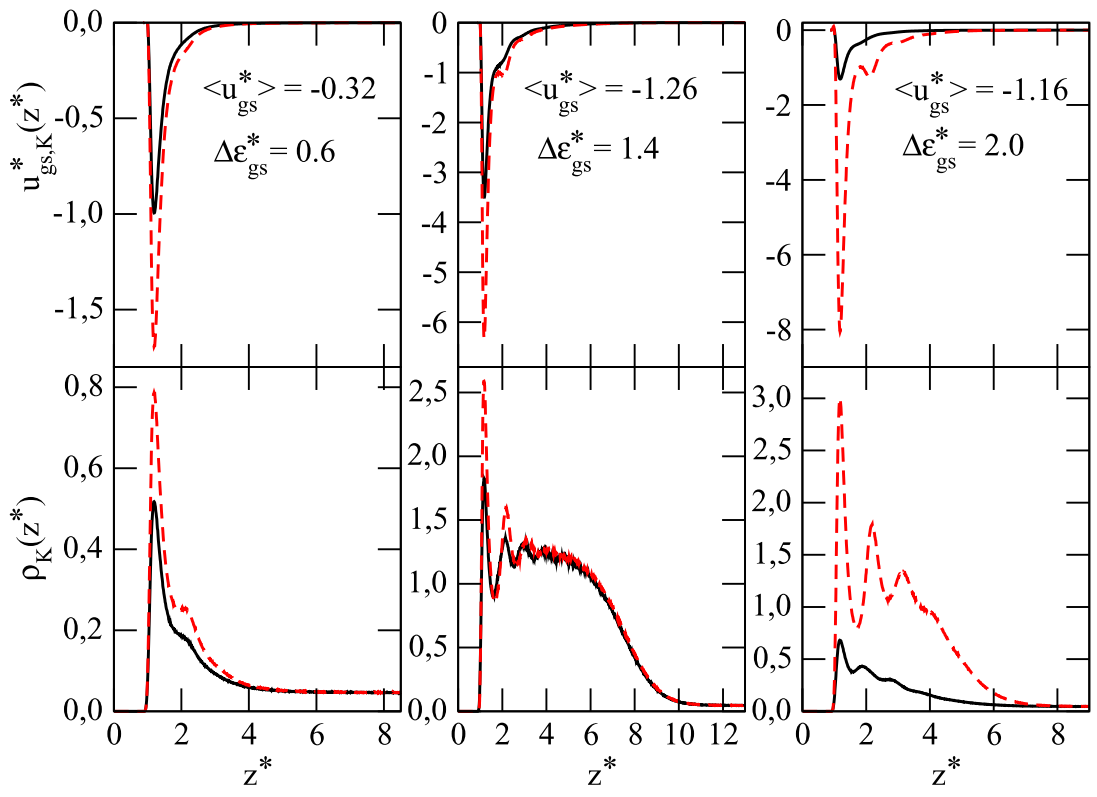

Fig. 11. The fluid-surface interaction energy profiles (upper panels), and the density profiles (lower panels), recorded at $T^{*}=0.92$ and $\mu^{*}=-3.75$, for the systems characterized by different values of $\Delta \varepsilon_{g s}^{*}$ given in the upper panels. In the upper panels, we have also given the average values of the fluid-surface potential energies, calculated under the same thermodynamic conditions.

However, the difference between the fluid-solid interaction in the first layer is greater since the component B interacts with the surface much more strongly than the component A. However, the density of component $\mathrm{A}$ is large even in the first layer, indicating a high degree of mixing. In a mixed layer, the number of particles interacting with the surface is greater than in the demixed one. In the dense layer, the average distance between a pair of BB (or AA) particles is slightly larger than $\sigma$, while the average distance between a pair of AB particles is lower, and slightly larger than $s \sigma$. In the consequence, a large number of interacting particles cause that the average fluid-surface potential energy is also large. The situation changes again when $\Delta \varepsilon_{g s}^{*}=2.0$. This system has the wetting temperature of about 0.925 . A large difference between $\varepsilon_{g s, A}^{*}$ and $\varepsilon_{g s, B}^{*}$ causes that at $T^{*}=0.92$ the film is highly demixed and remains stable up to the bulk coexistence. The total density of the first layer is, however, lower than in the case of $\Delta \varepsilon_{g s}^{*}=1.4$. This is due to the fact that this layer is predominantly occupied by the component $\mathrm{B}$, and hence, there are more $\mathrm{BB}$ and $\mathrm{AB}$ pairs. A small density of the component $\mathrm{A}$ in the film causes that its contribution to the fluid-solid interaction is low. Despite the fact that the component B shows a considerably stronger interaction with the surface than in the system with $\Delta \varepsilon_{g s}^{*}=1.4$, the total average fluid-surface potential energy is lower than in the system with $\Delta \varepsilon_{g s}^{*}=1.4$.

We have also performed similar simulations for the systems with $\bar{\varepsilon}_{g s}^{*}=6.0$ and different values of $\Delta \varepsilon_{g s}^{*}$, up to 3 , as well as for the systems with $\varepsilon_{g s, A}^{*}=6.0$ and the greater values of $\varepsilon_{g s, B}^{*}$, up to 9.0 . We recall that the mixture with $e=0.6$ and $s=0.7$ in contact with a non-selective wall characterized by $\varepsilon_{g s}^{*}=6.0$ has shown the triple point wetting at $T_{w}^{*}=T_{t r, v-m l-m s}^{*} \approx 0.875$. The systems with $\bar{\varepsilon}_{g s}^{*}=6.0$ have been found to behave similarly as the discussed earlier systems with $\bar{\varepsilon}_{g s}^{*}=5.5$. For small values of $\Delta \varepsilon_{g s}^{*}$, up to about 0.6 , the triple point wetting at $T_{w}^{*} \approx 0.875$ has been observed. A further increase of $\Delta \varepsilon_{g s}^{*}$ has caused the increase of the wetting temperature. When $\Delta \varepsilon_{g s}^{*}=1.0,2.0$ and 3.0, the wetting temperature has been estimated as equal to $0.89 \pm 0.005,0.925 \pm 0.005$ and $0.98 \pm 0.01$, respectively.

The behavior of the systems with $\varepsilon_{g s, A}^{*}=6.0$ and different values of $\Delta \varepsilon_{g s}^{*}$ has been found to be quite different than observed for the series with $\varepsilon_{g s, A}^{*}=5.0$. In the case of the series with $\varepsilon_{g s, A}^{*}=5.0$, the wetting transition at the non-selective wall occurs at $T_{w}^{*}=0.97 \pm 0.05$, i.e., well above the triple point $T_{w}^{*}=T_{t r, v-m l-m s}^{*}$. By increasing $\Delta \varepsilon_{g s}^{*}$, up to about 1.4 , the wetting temperature decreases to about 0.915 , and then starts to increase again, when $\Delta \varepsilon_{g s}^{*}$ becomes higher (cf. Fig. 10). When $\varepsilon_{g s, A}^{*}=6.0$, the increase of $\Delta \varepsilon_{g s}^{*}$, up to 3.0, has not been found to cause any changes of the wetting temperature. In all cases, we have observed the triple point wetting at $T_{w}^{*}=T_{t r, v-m l-m s}^{*}$. The wetting occurs via the first-order transition and is accompanied by the prewetting transition. For small values of $\Delta \varepsilon_{g s}^{*}$, up to about 0.6 , the onset of the prewetting transition is located right at the triple point $T_{t r, v-m l-m s}^{*}$. However, for the larger values of $\Delta \varepsilon_{g s}^{*}$, the onset of the prewetting transition occurs at lower temperatures (see Fig. 12). It does not imply that wetting occurs below the triple point. In the case of a strongly adsorbing non-selective walls, we have also observed the prewetting transitions to occur below the triple point [32], in agreement with the theoretical predictions of Pandit and Fisher [39]. The behavior observed agrees with the results obtained for the systems with non-selective walls discussed in reference [32]. Namely, it has been shown that 


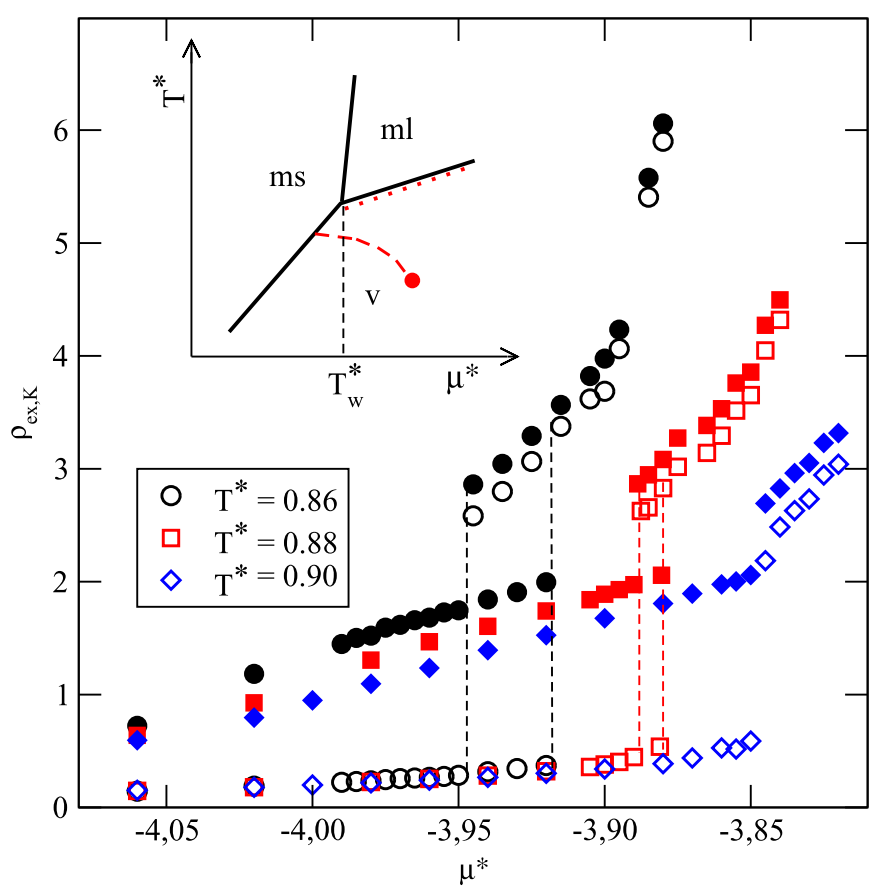

Fig. 12. The main figure shows the excess adsorption isotherms $\rho_{e x, K}$ of the components $K=A$ (open symbols) and $K=B$ (filled symbols) for the system with $\varepsilon_{g s, A}^{*}=6.0$ and $\Delta \varepsilon_{g s}^{*}=1.0$ at different temperatures. The inset shows the schematic representation of the phase diagram for the same system.

when the strength of the surface potential, $\varepsilon_{g s}^{*}$, exceeds 6.0 , the wetting transition is pinned in the triple point $T_{t r, v-m l-m s}^{*}$.

Now, we turn to the discussion of the wetting behavior of the mixture characterized by $s=0.6$ and $s=0.72$. This mixture has the triple point $T_{t r, v-m l-m s}^{*}$ at the temperature equal to $0.835 \pm 0.005$ and the triple point $T_{t r, v-m l-d l}^{*}$ at $0.97 \pm 0.005$. We have considered two series of systems, in which $\varepsilon_{g s, A}^{*}=5.0$ and 6.0 , while $\varepsilon_{g s, B}^{*}$ has been assumed to be larger and changed between 5.2 and 6 . In the first case of $\varepsilon_{g s, A}^{*}=5.0$, the wetting transition at the non-selective wall has been found to occur right at the temperature of the triple point $T_{t r, v-m l-d l}^{*}$, i.e., at $T *_{w}=0.97 \pm 0.005$. Thus, only the demixed film wets the surface. On the other hand, when $\varepsilon_{g s, A}^{*}=6.0$, the wetting transition has been estimated to occur at a considerably lower temperature, at $T_{w}^{*}=0.875 \pm 0.005$ [32].

In the case of the series with $\varepsilon_{g s, A}^{*}=5.0$, one can expect that the wall selectivity should not lead to the changes of the wetting temperature. An increase of $\Delta \varepsilon_{g s}^{*}$ is bound to enhance demixing in the film. Therefore, at the temperatures below the triple point $T_{t r, v-m l-d l}^{*}$, the film is likely to remain demixed up to the bulk coexistence, and hence cannot wet the surface, since the bulk liquid is mixed. On the other hand, as soon as the temperature reaches or exceeds the triple point $T_{t r, v-m l-d l}^{*}$, the enhanced demixing in the film favors the formation of thick wetting layer, since the bulk liquid is also demixed. Figures 13 and 14 present

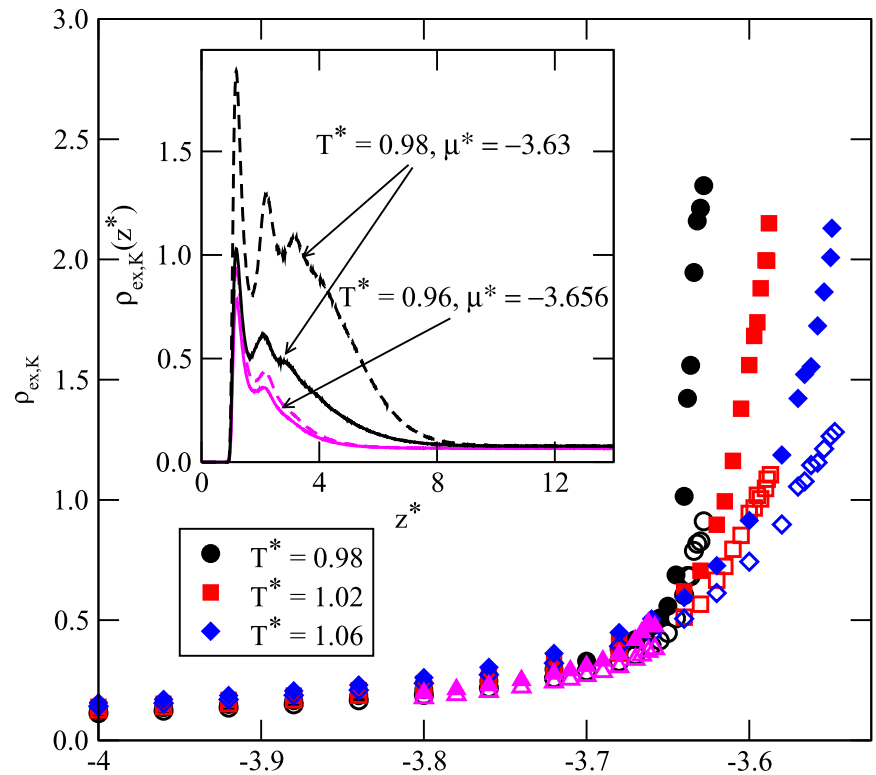

Fig. 13. The excess adsorption isotherms of individual components for the system with $s=0.72, \varepsilon_{g s, A}^{*}=5.0$, and $\varepsilon_{g s, B}^{*}=5.2$. The inset shows the examples of density profiles of the components A (solid lines) and B (dashed lines) at the temperature below and above the wetting temperature. The estimated values of the chemical potential at the bulk coexistence are equal to about $\mu_{o}^{*}=-3.65$ and -3.627 at $T^{*}=0.96$ and 0.98 , respectively.

the examples of the excess adsorption isotherms and density profiles, obtained for the systems with $\Delta \varepsilon_{g s}^{*}=0.2$ and 1.0 , respectively. When $\Delta \varepsilon_{g s}^{*}=0.2$, the adsorption of both components remain quite low as long as the temperature is lower than $T_{t r, v-m l-d l}^{*}$. The density profiles calculated at $T^{*}=0.96$ and 0.98 , and for the chemical potential values very close to the bulk coexistence (see the inset to Fig. 13) demonstrate that at the temperature below the triple point $(0.96)$, the film thin and shows a low degree of demixing. On the other hand, at $T^{*}=0.98$, the developing film is highly demixed and attains much higher thickness. The same occurs at still higher temperatures.

When the value of $\varepsilon_{g s, B}^{*}$ increases, the predominant adsorption of component $\mathrm{B}$ also increases and hence the adsorbed film can reach greater thickness, even at the temperatures below the wetting transition. This is illustrated by the excess adsorption isotherm at $T^{*}=0.96$, shown in the main part of Figure 14. At the temperatures above $T_{w}^{*}$, the film thickness increases, although the density profiles of individual components look quite similar. It should be noted that the wetting is the first order transition, and one should not expect the divergence of the film thickness, as long as the chemical potential is lower than the bulk coexistence value.

The calculations have demonstrated that the systems discussed exhibit the prewetting transition already below the wetting temperature. The onset of the prewetting transitions have been found to occur at gradually decreasing temperatures when $\Delta \varepsilon_{g s}^{*}$ increases. This demonstrates that the phase diagrams for these systems look like that 


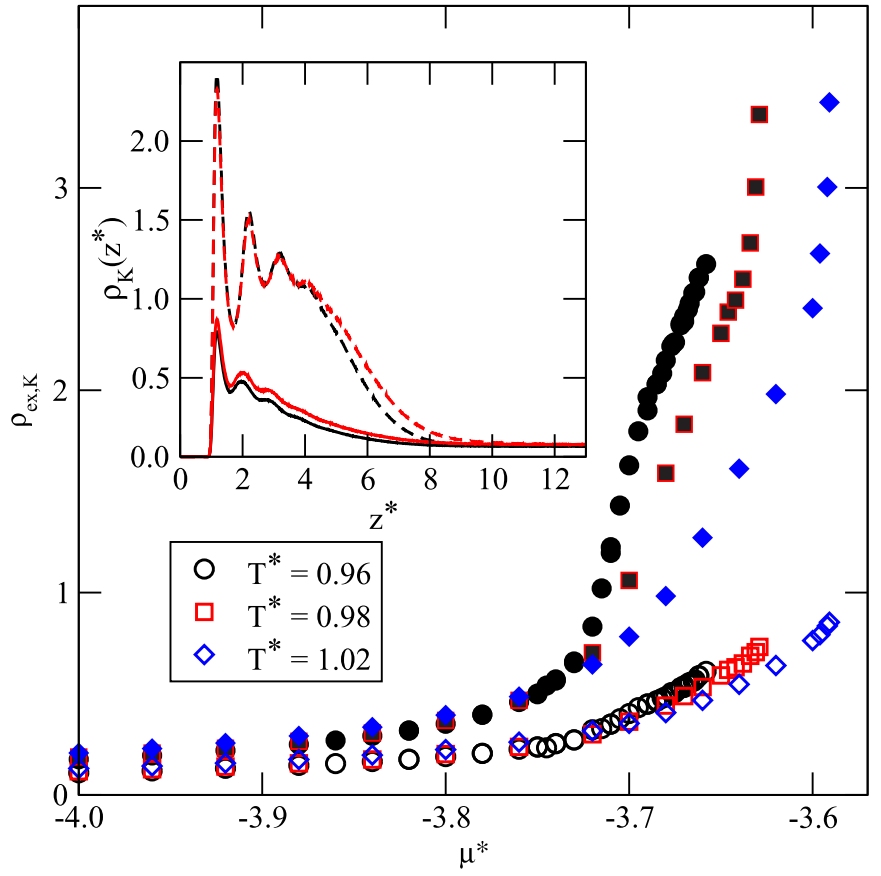

Fig. 14. The excess adsorption isotherms of individual components for the system with $s=0.72, \varepsilon_{g s, A}^{*}=5.0$ and $\varepsilon_{g s, B}^{*}=6.0$. The inset shows the examples of density profiles of the components A (solid lines) and B (dashed lines) at the temperature below and above the wetting temperature. The profiles at $T^{*}=0.96$ and 0.98 have been recorded for $\mu^{*}=-3.658$ and -3.629 , respectively.

given in the inset of Figure 12, but with the triple point $T_{t r, v-m l-m s}^{*}$ replaced by the triple point $T_{t r, v-m l-m s}^{*}$ and with the condensed phases $\mathrm{ms}$ and $\mathrm{ml}$ replaced by $\mathrm{ml}$ and $\mathrm{dl}$, respectively.

The series of systems characterized by $e=0.6, s=0.72$, $\varepsilon_{g s, A}^{*}=6.0$ and different values of $\varepsilon_{g s, B}^{*}$ between 6.2 and 7.0 have shown still different wetting behavior. For the values of $\Delta \varepsilon_{g s}^{*}<0.6$, the wetting temperature has been found to decrease from about 0.875 , when $\Delta \varepsilon_{q s}^{*}=0$ to 0.835, when $\Delta \varepsilon_{g s}^{*}=0.6$. A further increase of $\Delta \varepsilon_{g s}^{*}$ up to 1.0 has not led to any changes of the wetting temperature. We recall that the triple point $T_{t r, v-m l-m s}^{*}$ is located just at the temperature of 0.835 , so that the triple point wetting occurs for large values of $\Delta \varepsilon_{g s}^{*}$. Although, we have not carried out calculations for the values of $\Delta \varepsilon_{g s}^{*}$ greater than 1.0, nevertheless it can be anticipated that the wetting temperature should gradually increase when $\Delta \varepsilon_{g s}^{*}$ becomes high enough. This prediction is based on the results obtained for the the previously discussed systems with $s=0.7$ and $\varepsilon_{g s, A}^{*}=6.0$, as well as on the results obtained for the system with $s=0.73$ and $\varepsilon_{g s, A}^{*}=6.0$ [35]. There is, however, a difference in the behavior of the systems with $s=0.7,0.72$ and 0.73 , resulting from a different tendency towards demixing. Figure 15 shows the plots of the wetting temperature against $\Delta \varepsilon_{g s}^{*}$ the systems with different $s$. In the case of $s=0.7$ and $\varepsilon_{g s, A}^{*}=6$, the surface field is strong enough to ensure a complete wetting already at the triple point $T_{t r, v-m l-m s}^{*}$. An increase of $\Delta \varepsilon_{g s}^{*}$ causes that the reentrant mixing in the film occurs

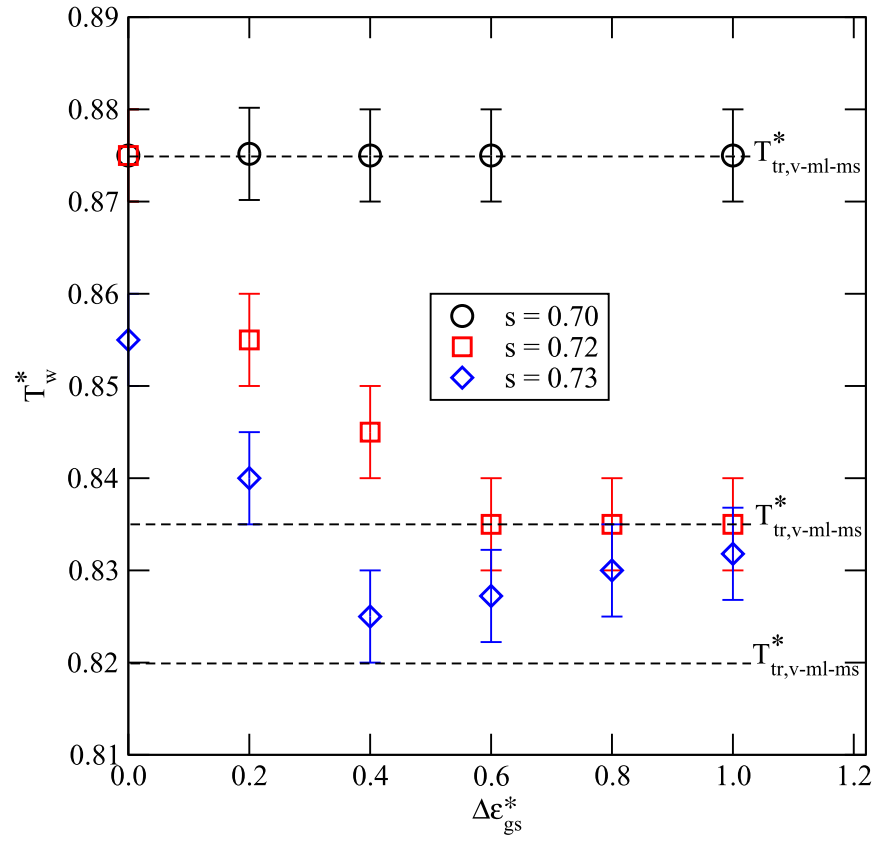

Fig. 15. The plots of the wetting temperature versus $\Delta \varepsilon_{g s}^{*}$, for the systems with $e=0.6, \varepsilon_{g s, A}^{*}=6.0$ and different values of $s$ (shown in the figure). The horizontal dashed lines mark the locations of the triple point $T_{t r, v-m l-m s}^{*}$ in the bulk mixtures of different values of the parameter $s$.

even when the component B shows a strong preferential adsorption. The solid phase does not wet the surface, and it results in the pinning of the wetting transition in the triple point. For the larger values of $s=0.72$ and 0.73 , the situation changes. When the surface is non-selective, the wetting transition occurs above the corresponding triple points. The increase of $\varepsilon_{g s, B}^{*}$ leads to stronger average fluid-solid interaction, and moves the wetting transition towards lower temperatures. In the case of $s=0.72$, the wetting temperature reaches the triple point $T_{t r, v-m l-m s}^{*}$, when $\Delta \varepsilon_{g s}^{*} \approx 0.6$. For still higher values of $\Delta \varepsilon_{g s}^{*}$ the triple point wetting occurs. The mixture with $s=0.72$ has a larger tendency towards demixing than the mixture with $s=0.7$, and hence the reentrant mixing should also be suppressed when $\Delta \varepsilon_{g s}^{*}$ becomes sufficiently high. However, this phenomenon has not been observed for the largest value of $\Delta \varepsilon_{g s}^{*}=1.0$ considered here. The suppression of reentrant mixing occurs in the mixture with $s=0.73$, which exhibits still higher tendency towards demixing [35], and results in a gradual increase of the wetting temperature when $\Delta \varepsilon_{g s}^{*}$ is higher than about 0.4.

\section{Summary}

In this paper, we have attempted to elucidate the interplay between the properties of bulk highly non-additive symmetric mixtures, with the parameter $e=0.6$, and their wetting behavior at selective walls. To this end, we have performed extensive Monte Carlo study in the grand canonical ensemble. The mixtures considered are characterized by different value of the parameter $s$, equal 
to 0.70 and 0.72 , and exhibit the closed immiscibility loops. The demixing transition along the vapor-liquid coexistence occurs only at sufficiently high temperatures, above the triple point $T_{t r, v-m l-d l}^{*}$, in which the vapor coexists with the mixed and demixed liquids. At lower temperatures, below $T_{t r, v-m l-d l}^{*}$, but above the triple point $T_{t r, v-m l-m s}^{*} \approx 0.815$, in which the vapor, the mixed liquid and the mixed solid coexist, the vapor condenses into the mixed liquid. The bulk phase diagrams of the mixtures studied are qualitatively the same (cf. Fig. 1), but they show different tendency towards demixing, resulting from the changes of the geometrical non-additivity, measured by the magnitude of the parameter $s$. In the case of $s=0.7$, the vapor condenses into the demixed liquid only over a very narrow range of temperatures, between $T_{t r, v-m l-d l}^{*} \approx 1.11$ and $T_{t r c, 2}^{*} \approx 1.18$. On the other hand, the mixture with $s=0.72$ exhibits a considerably higher tendency towards demixing, and the vapor condensation leads to the formation of demixed liquid phases already at $T_{t r, v-m l-d l}^{*} \approx 0.97$. The tricritical point $T_{t r c, 2}^{*}$ is located between 1.18 and 1.175 .

We have considered the systems with the fixed average value of, $\bar{\varepsilon}_{g s}^{*}=0.5\left(\varepsilon_{g s, A}^{*}+\varepsilon_{g s, B}^{*}\right)$, as well as the systems in which $\varepsilon_{g s, A}^{*}$ has been fixed, while $\varepsilon_{g s, B}^{*}$ assumed different, greater than $\varepsilon_{g s, A}^{*}$, values.

In the case of the fixed average values of $\bar{\varepsilon}_{g s}^{*}$, equal to 5.5 and 6.0 , we have found that the wetting temperature does not change for $\Delta \varepsilon_{g s}^{*}$ lower than a certain threshold value. Then the wetting temperature rather sharply increases with $\Delta \varepsilon_{g s}^{*}$. This behavior can be understood by taking into account that small values of $\Delta \varepsilon_{g s}^{*}$ considerably affect only the composition of the layers adjacent to the surface, so that the developing film can readily undergo the reentrant mixing. Since the value of $\bar{\varepsilon}_{g s}^{*}$ is constant, the average fluid-surface interaction in the thick films is practically unaffected by the behavior of the layers adjacent to the surface, and hence, the wetting temperature does not change. Once the value of $\Delta \varepsilon_{g s}^{*}$ becomes larger than the threshold value, the stability of thin demixed layer becomes sufficiently large to hinder the reentrant mixing, and the demixed film survives even when the chemical potential reaches the bulk condensation point. Only at sufficiently high temperatures, the increasing entropic effects allow for the reentrant mixing to occur. This leads to large increase of the wetting temperature.

The behavior of systems with the fixed value of $\varepsilon_{g s, A}^{*}$ and different (higher) values of $\varepsilon_{g s, B}^{*}$ has been found to considerably different. In such cases, the increase of $\Delta \varepsilon_{g s}^{*}$ leads to the increase of the average fluid-solid interaction. Therefore, one expects a gradual lowering of the wetting temperature with $\Delta \varepsilon_{g s}^{*}$. We have found that it happens only when the wetting transition at non-selective wall occurs above the triple point $T_{t r, v-m l-m s}^{*}$, in which the vapor coexists with the mixed liquid and solid phases, and for small values of $\Delta \varepsilon_{g s}^{*}$ (cf. Figs. 10 and 15). In the system with $s=0.72$ and $\varepsilon_{g s, A}^{*}=6.0$, the wetting temperature has been found to decrease and reach the triple point $T_{t r, v-m l-m s}^{*}$ for $\Delta \varepsilon_{g s}^{*} \approx 0.6$. A further increase of $\Delta \varepsilon_{g s}^{*}$ to 1.0 has not changed the wetting temperature. In the case when the wetting at a non-selective wall occurs right in the triple point $T_{t r, v-m l-m s}^{*}$, an increase of $\Delta \varepsilon_{g s}^{*}$ does not change the wetting temperature. This implies that the solid phase does not wet the surface.

The results presented above have demonstrated that the wetting behavior of highly non-additive symmetric mixture strongly depends on the properties of the bulk mixtures as well as on the strength of interactions between the mixture components and the surface. When the wetting transition occurs at non-selective walls occurs above the triple point $T_{t r, v-m l-m s}^{*}$ and below the triple point $T_{t r, v-m l-d l}^{*}$, weakly selective walls have been found to lead to a decrease of the wetting temperature. On the other hand, strongly selective walls may cause an increase of the wetting temperature, due to enhance stability of demixed films. In the case of triple point wetting, in $T_{t r, v-m l-m s}^{*}$, at non-selective walls, the wall selectivity does not lead to the decrease of the wetting temperature, since the solid phase does not wet the surface.

Open Access This is an open access article distributed under the terms of the Creative Commons Attribution License (http://creativecommons.org/licenses/by/4.0), which permits unrestricted use, distribution, and reproduction in any medium, provided the original work is properly cited.

\section{References}

1. N.B. Wilding, F. Schmid, P. Nielaba, Phys. Rev. E 58, 2201 (1998)

2. S. Materniak, A. Patrykiejew, S. Sokołowski, J. Chem. Phys. 133, 244501 (2010)

3. A. Patrykiejew, S. Sokołowski, Phys. Rev. E 81, 012501 (2010)

4. S. Materniak, A. Patrykiejew, W. Rżysko, Phys. Rev. E 87, 062306 (2013)

5. D. Wojwod, M. Schoen, Phys. Rev. E 73, 011201 (2006)

6. N.B. Wilding, Phys. Rev. E 67, 052503 (2003)

7. M.J. Vlot, C. Classen, H.E.A. Huitema, J.P. Van der Eergen, Mol. Phys. 91, 19 (1997)

8. A. Patrykiejew, Phys. Rev. E 95, 012145 (2017)

9. J.R. Silbermann, W. Woywod, M. Schoen, Phys. Rev. E 69, 031606 (2004)

10. A. Patrykiejew, L. Sałamacha, S. Sokołowski, Phys. Rev. E 67, 061603 (2003)

11. E. Kirlik, M.L. Rosinberg, P.A. Monson, J. Chem. Phys. 101, 10947 (1994)

12. K. Bucior, A.Patrykiejew, O. Pizio, S. Sokołowski, Mol. Phys. 101, 1477 (2003)

13. D. Woywod, M. Schoen, J. Phys.: Condens. Matter 14, $4761(2004)$

14. F. Schmid, N.B. Wilding, Phys. Rev. E 63, 031201 (2001)

15. N.B. Wilding, F. Schmid, Comp. Phys. Commun. 147, 149 (2002)

16. N.B. Wilding, M. Krech, Phys. Rev. E 57, 5795 (1998)

17. E. Kirlik, Y. Fan, P.A. Monson, M.L. Rosinberg, J. Chem. Phys. 102, 3712 (1995)

18. Y. Rouault, J. Baschnagel, K. Binder, J. Stat. Phys. 80, 1009 (1995)

19. E. Paschinger, D. Levesque, G. Kahl, J.-J. Weis, Europhys. Lett. 55, 178 (2001) 
20. E. Schöl-Paschinger, D. Levesque, J.-J. Weis, G. Kahl, Phys. Rev. E 64, 011502 (2001)

21. D. Woywod, M. Schoen, Phys. Rev. E 67, 026122 (2003)

22. K. Bucior, A. Patrykiejew, O. Pizio, S. Sokołowski, Z. Sokołowska, Mol. Phys. 101, 721 (2003)

23. A. Martinez, A. Patrykiejew, O. Pizio, S. Sokołowski, J. Phys.: Condens. Matter 153107 (2003)

24. A. Patrykiejew, O. Pizio, S. Sokołowski, J. Phys. Chem. B 109, $14227(2005)$

25. J. Jacques, A. Collet, S.H. Wilen, Enantiomers, Racemates, and Resolutions (Krieger, Melbourne, FL, 1991)

26. B. Waldect, Chirality 5, 350 (1993)

27. V. Schurig, H.-P. Novotny, J. Chromatogr. 441, 155 (1988)

28. R.M. Hazen, D.S. Sholl, Nat. Mater. 2, 367 (2003)

29. J.D. Horvath, A.J. Gellman, Top. Catal. 25, 9 (2003)
30. A.J. Gellman, Y. Huang, X. Feng, V.V. Pushkarev, B. Holsclaw, B.S. Mhatre, J. Am. Chem. Soc. 135, 19208 (2013)

31. A. Patrykiejew, J. Stat. Mech. 2017, 123208 (2017)

32. A. Patrykiejew, Phys. Chem. Chem. Phys. 20, 9228 (2018)

33. J.W. Cahn, J. Chem. Phys. 663667 (1977)

34. C. Ebner, W.F. Saam, Phys. Rev. Lett 38, 1486 (1977)

35. A. Patrykiejew, Condens. Matter Phys. 21, 23602 (2018)

36. D. Frenkel, B. Smit, Understanding Molecular Simulation: From Algorithms to Applications (Academic Press, San Diego, 1996)

37. M.P. Allen, D.J. Tildesley, Computer Simulation of Liquids (Oxford University Press, Oxford, 1987)

38. D.P. Landau, K. Binder, A Guide to Monte Carlo Simulation in Statistical Physics (Cambridge University Press, Cambridge, 2000)

39. R. Pandit, M. Fisher, Phys. Rev. Lett. 51, 1772 (1983) 